\title{
Videothoracoscopic identification of chondromatous hamartoma of the lung
}

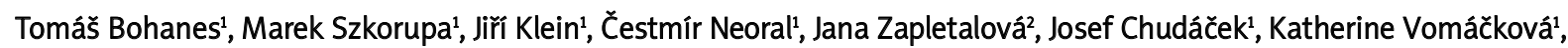 \\ Radek Vrba ${ }^{1}$ \\ ${ }^{1}$ The First Department of Surgery, Palacký University Teaching Hospital, Olomouc, Czech Republic \\ 2Department of Medical Biophysics, Palacký University Faculty of Medicine and Dentistry, Olomouc, Czech Republic
}

Videosurgery Miniinv 2013; 8 (2): 152-157

DOI: 10.5114/wiitm.2011.33013

\begin{abstract}
Introduction: The main disadvantage of a videothoracoscopic procedure is the lack of touch sensation. The probability of easily finding the lesion is usually estimated according to computed tomography (CT).

Aim: To find useful parameters of location of chondromatous hamartoma of the lung parenchyma in relation to its size to assess the probability of successful search via a videothoracoscopic approach only.

Material and methods: A group of 55 patients operated on for chondromatous hamartoma of the lung at the First Department of Surgery in Olomouc from January 2006 to June 2011 was analyzed. Initially, the tumor's longest diameter and its nearest distance to the pleural surface were measured on CT scans. Subsequently, the surgery began using the videothoracoscopic approach. A short thoracotomy with direct palpation followed when videothoracoscopy failed. Results: No significant differences in age, sex and side of localization between the group with and without successful videothoracoscopic detection were found. A significant difference was found in the median size $(p=0.026)$ and the depth of the tumor $(p<0.0001)$ and in the calculated index "tumor size/depth" $(p<0.0001)$. Deeper analysis revealed that the parameters "depth" and "index size/depth" are considered to be good predictors but the parameter "size" is not a suitable predictor.

Conclusions: The main predictors of successful videothoracoscopic detection of lung chondromatous hamartoma are considered to be the depth of the tumor in the lung parenchyma with a cut-off value $\leq 7.5 \mathrm{~mm}$ and the index "size/depth" with a cut-off value $\geq 1.54$; the tumor size is not considered to be a good predictor.
\end{abstract}

Key words: chondromatous hamartoma, lung, videothoracoscopy, video-assisted thoracic surgery, pulmonary nodule.

\section{Introduction}

The technique of thoracoscopy was introduced by Jacobaeus in 1910, primarily for the diagnostics of pleural and pulmonary diseases [1]. Since that time significant advances in surgical equipment, endoscopic stapling devices, video monitoring and endoscopic surgical techniques have expanded the role of thoracoscopy in many intrathoracic pathologic conditions where it plays a very important role [2-6]. The benefit of this minimally invasive approach for thoracic surgery patients is obvious; reduction of pain and cosmetic effect are the most frequently reported improvements $[7,8]$. On the other hand, the aforementioned pain relief remains controversial according to other studies [9].

The main disadvantage of such a videothoracoscopic procedure is the lack of touch sensation during 
the procedure. However, direct palpation in detection of occult nodules in the lung parenchyma can be vital, especially when lesions are located deeper in the tissue and they are smaller. In almost all guidelines and textbook descriptions of video-assisted thoracic surgery procedures (VATS) for lung nodules, direct palpation is considered to be an essential element of the procedure, but it requires a short thoracotomy for insertion of one or several fingers into the patient's chest. This is certainly more invasive than simple videothoracoscopy with only punctures for insertion of trocars and it is against the contemporary trend to reduce the surgery trauma as much as possible [10]. That is why several methods of marking the nodule site for a subsequent videothoracoscopic procedure were introduced, e.g. injection of dye [11] or application of a hook wire under computed tomography (CT) control [12] or perioperative ultrasonography [13].

Among typical indications for such a minimally invasive procedure in the chest are chondromatous hamartomas. Pulmonary chondromatous hamartoma $(P C H)$ is defined as a benign tumor which originates from the mesenchymal cells associated with the bronchial and bronchiolar walls. It is composed of mature and immature chondroid tissue, adipose tissue, osteoid and smooth muscle cells in various amounts and ratios. Epithelial elements such as bronchial and bronchiolar epithelium, type II pneumocytes and rarely bronchiolar glands may also be present in chondromatous hamartomas [14-16]. A pathogenetic relationship between $\mathrm{PCH}$ and lipoma and leiomyoma is obvious whereas the progenitor mesenchymal cell situated in the bronchial wall may differentiate along chondroid, adipose and smooth muscle. It has also been demonstrated that epithelial proliferation in $\mathrm{PCH}$ is reactive and closely associated with neoplastic proliferation of the mesenchymal component [16].

Lung hamartomas are generally indicated for removal. Primarily, sarcomatous transformation in a long-standing, clinically silent hamartoma has been reported [17]. Secondly, it may sometimes be difficult to differentiate benign hamartomas from other primary and secondary tumors of the lung or other lesions such as tuberculoma etc. solely according to imaging examinations. Moreover, it is usually relatively easy to find and enucleate these cartilaginous rounded masses from the lung parenchyma with minimal injury to the tissue. In most cases, it may be done employing only the minimally invasive approach provided that we can find the mass in the lung parenchyma without direct palpation of the lung.

The probability of finding the lesion without direct touch is usually estimated by a surgeon according to the evaluation of CT findings. Nonetheless, this kind of estimation is very subjective and requires extensive experience.

\section{Material and methods}

To try to find a more objective approach to the issue, the authors prospectively studied a group of patients operated on for this diagnosis at the First Department of Surgery, Palacký University Hospital Olomouc from January 2006 to June 2011. A standard protocol was used in all of these patients. Patients with suspicion of chondromatous hamartoma were indicated for surgery. Prior to the operation, the tumors were measured on CT scans and the longest diameter and nearest distance to the pleural surface were recorded.

The surgery always began using the videothoracoscopic approach and was performed under selective ventilation of the contralateral lung. Trocar placement was determined according to location of the tumor. A surgeon revised the pleural cavity to exclude other pathology and then tried to locate the tumor by inspection and with "palpation" using an endoscopic instrument with or without a tampon. In the hands of an experienced thoracic surgeon, this "palpation" method was able to reveal deeply located tumors within the collapsed lung. When the tumor was located, its enucleation via the videothoracoscopic approach followed with cutting of the visceral pleura and parenchyma and removal of the tumor through this pulmotomy, which was finally sutured with a stitch. When videothoracoscopic search with instrument-only "palpation" failed, a short thoracotomy was performed by enlarging one of the previous incisions so that it would be possible to insert one or more fingers into the pleural cavity for direct palpation of the lung tissue. In several patients perioperative ultrasound was employed in case of failed simple videothoracoscopic search as part of another research, but this did not influence the results of the described study. After tumor detection, surgery followed as in the previous case, unless the tumor was located too deep or in the vicinity of some main 
vessels. In this situation it was necessary to finish the procedure through a short thoracotomy. Five patients were excluded from the study group when the perioperative finding was different from the presupposed diagnosis of chondromatous hamartoma (carcinoid, tuberculoma, metastasis) because of theoretically different touch feeling in these nodules (they are usually softer and less circumscribed, which may bias the results) and those procedures were finished according to the finding.

The obtained data of preoperative measurements and results of detection during the procedure were analyzed statistically. The goal was to find parameters of tumor location within the lung parenchyma in relation to its size allowing assessment of the probability of successful search of the tumor via the videothoracoscopic approach without the need to enlarge the incision and with no special technique employed for its detection. The data were statistically analyzed

Table I. Characteristics of the group of patients

\begin{tabular}{|lccc|}
\hline Variable & TS $+(n=26)$ & TS $-(n=29)$ & Value of $p$ \\
\hline Age & $\begin{array}{c}54.5 \\
(48.5-59.8)\end{array}$ & $\begin{array}{c}56.0 \\
(50.0-65.0)\end{array}$ & $0.637 \mathrm{a}$ \\
\hline Sex (M/F) & $\begin{array}{c}14 / 12 \\
(53.8 \% / 46.2 \%)\end{array}$ & $\begin{array}{c}15 / 14 \\
(51.7 \% / 48.3 \%)\end{array}$ & $0.875 \mathrm{~b}$ \\
\hline Side (L/R) & $\begin{array}{c}9 / 17 \\
(34.6 \% / 65.4 \%)\end{array}$ & $\begin{array}{c}10 / 19 \\
(34.5 \% / 65.5 \%)\end{array}$ & $0.992^{\mathrm{b}}$ \\
\hline
\end{tabular}

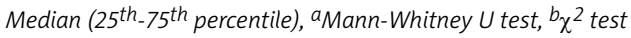

using the software SPSS version 15 (SPSS Inc., Chicago, USA). The categorical parameters were compared by means of the $\chi^{2}$ test or Fisher's exact test. The Mann-Whitney $U$ test was used to compare continuous parameters. The normality of distribution was checked by the Shapiro-Wilk test. Logistic regression analysis and receiver operating characteristics (ROC) analysis were used to identify suitable predictors of probability of videothoracoscopic detection of the tumor. All tests were performed at the significance level of 0.05 .

\section{Results}

During the study period, the conditions determined to be evaluated in the study were fulfilled in 55 patients ( 29 males, 26 females). No significant differences in age, sex and side of localization between the group with successful videothoracoscopic finding of the tumor and without were found (Table I).

The groups of patients were analyzed as to the location of the tumor in specific segments of the lungs. In both groups the most frequent occurrence was found in segment 3 (38.5\%/20.7\%). No significant difference was found using Fisher's exact test $(p=0.485)$ (Figure 1 ).

Analysis found statistically significant differences in the most expected values, i.e. in median tumor size, depth of the tumor location, and the index tumor size/depth of location, confirmed by the Mann-Whitney U test (Table II).

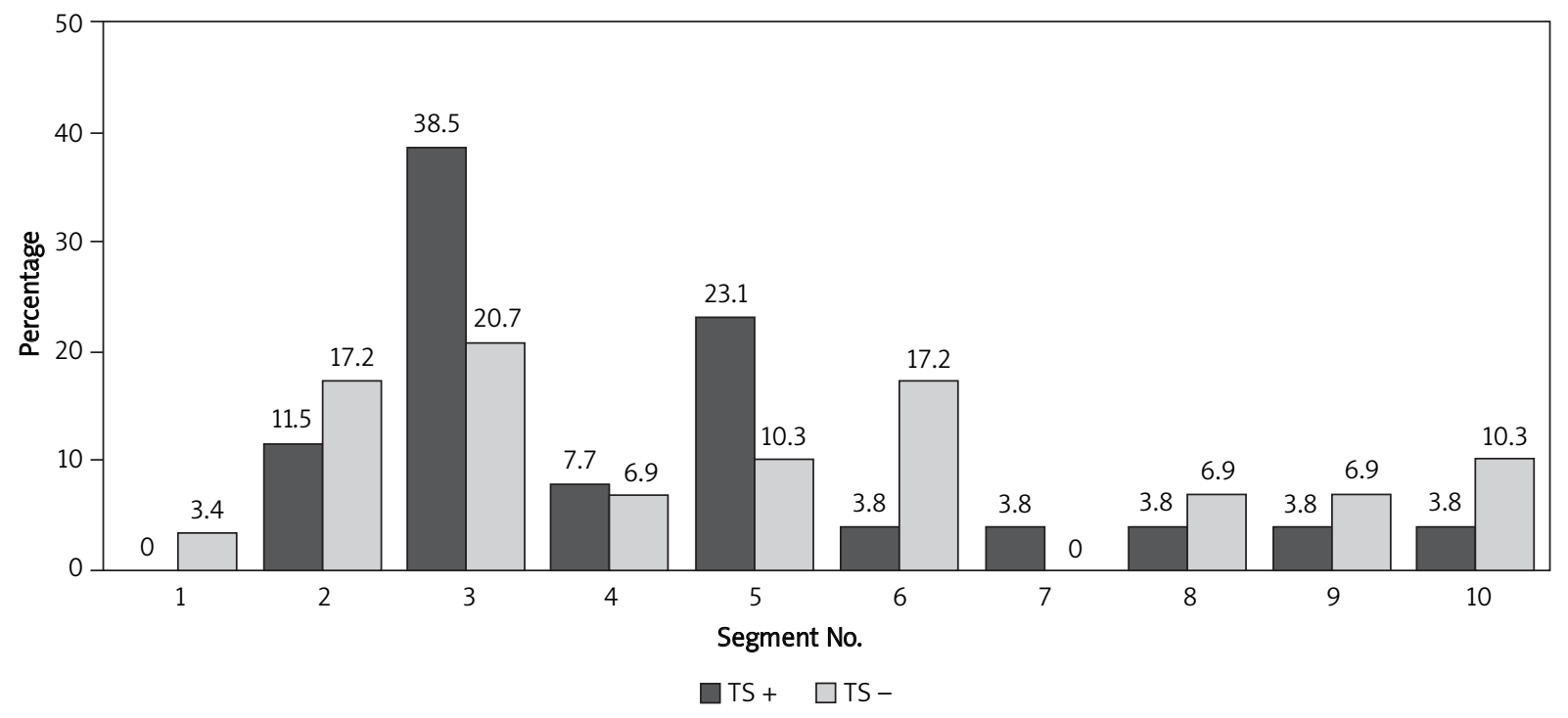

Figure 1. Distribution of the tumors in the lung segments 
The median size of the tumor in the detectable group was $15 \mathrm{~mm}$ (min-max: 8-45 $\mathrm{mm}$ ), in the undetectable group $10 \mathrm{~mm}(6-40 \mathrm{~mm})(p=0.026)$. The tumors in the detectable group were located more superficially, with median depth of $5 \mathrm{~mm}(0-10 \mathrm{~mm})$, than those in the undetectable group, with $12 \mathrm{~mm}$ (3-52 mm) $(p<0.0001)$. The index tumor size/depth was calculated and was different in the two groups $(3.4 / 0.8)(p<0.0001)$.

\section{Discussion}

The probability of successful videothoracoscopic detection of a lung lesion is certainly quite a complex matter. It is possible to employ various special preoperative or perioperative techniques which are able to facilitate the detection of an occult tumor. Nonetheless, this increases the costs and these techniques are also related to certain adverse effects. Therefore, it may be profitable to assess several known parameters to estimate the necessity of such a sophisticated approach. Measurement of two easily available distances is certainly a simple method, which may improve our knowledge before the start of the procedure. In spite of this fact, there are not many studies which address this issue and offer a clear solution.

Our results correspond well with the results of Tamura et al. [18], who found a similar relation of size and depth of location to probability of successful videothoracoscopic detection. However, if we analyze the date more closely, we can find that not all of these presented statistically significant results are good predictors for assessment of probability of successful detection of the tumor. Application of logistic regression analysis to our data shows that only the tumor size and index size/depth are suitable predictors in comparison to the size itself. Increase of the value of the index size/depth by 1 is related to a decrease in the chance of videothoracoscopy detecting the tumor 0.571 times $(95 \% \mathrm{Cl}$ for OR: $0.394-0.826, p=0.003)$. Increase of the tumor size by $1 \mathrm{~mm}$ means increase of the chance of detection 1.11 times (95\% Cl: 1.001-1.232, $p=0.048)$. Subsequently, this is confirmed by ROC analysis, which may also show us values of cut-off of optimal parameters for prediction of chance of videothoracoscopic detection of the tumor. The parameters "depth" and "index size/depth" are considered to be good predictors with values of AUC (area under ROC curve) of 0.899
Table II. Results of analysis of size and depth of the tumor and index size/depth

\begin{tabular}{|lccc|}
\hline Variable & TS $+(n=26)$ & TS $-(n=29)$ & Value of $p$ \\
\hline Size $[\mathrm{mm}]$ & $15.0(10.8-20.0)$ & $10.0(9.0-14.5)$ & $0.026^{\mathrm{a}}$ \\
\hline Depth $[\mathrm{mm}]$ & $5.0(0.0-6.0)$ & $12.0(8.5-22.5)$ & $<0.0001^{\mathrm{a}}$ \\
\hline Size/depth & $3.4(2.5-92.5)$ & $0.8(0.5-1.4)$ & $<0.0001^{\mathrm{a}}$ \\
\hline
\end{tabular}

Data are expressed as median (25th and $75^{\text {th }}$ percentile), Mann-Whitney $U$ test

and 0.931. In contrast, the parameter "size" is not a suitable predictor with AUC of 0.674 (Figure 2). Optimal cut-off values of the important predictors are shown in Table III. Similar cut-off values were presented by Tamura et al., with their values of $>10 \mathrm{~mm}$ in depth and $<15 \mathrm{~mm}$ in diameter [13], although they conclude the cut-off value as "no chance" whereas our conclusion represents values of borderline of good probability of successful detection.

A rather similar approach was presented by Demmy et al. [19], who created three formulas for their similar group of patients, which calculate potential "visibility", "respectability" and overall "simplicity" using the same data of depth and size of the tumor, where their "visibility" is almost identical to our "index size/depth". Our results show, however, that

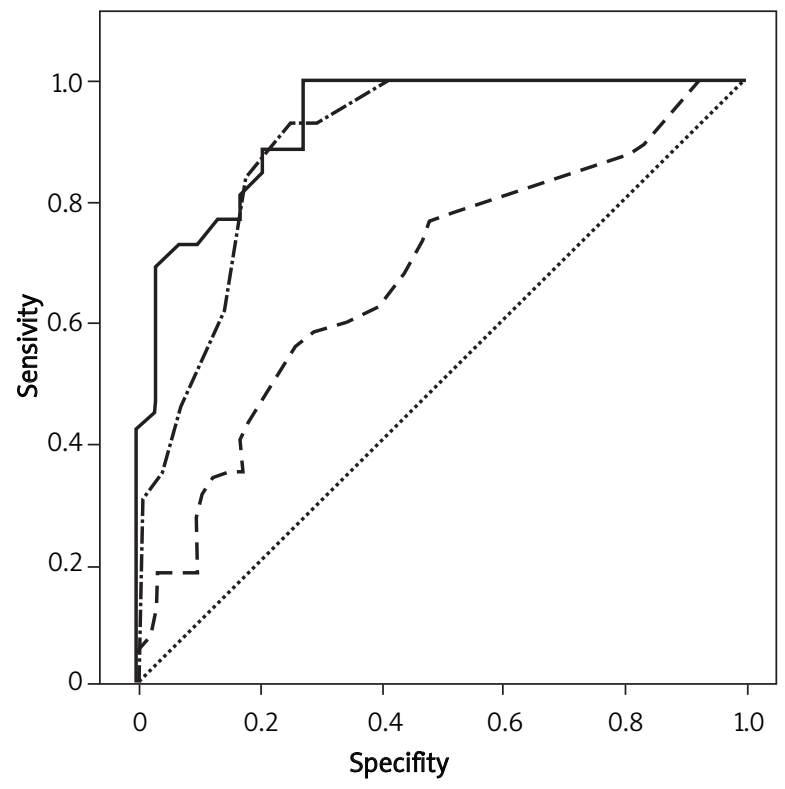

Source of curve:

- - Size — Size/depth -.-Depth ....... Reference line

Figure 2. ROC curve 
Table III. Optimal cut-off values of the main predictors

\begin{tabular}{|lccccc|}
\hline Variable & AUC & Cut-off value & Sensitivity [\%] & Specificity [\%] & Accuracy [\%] \\
\hline Depth of tumor location & 0.899 & $\leq 6.5 \mathrm{~mm}$ & 84.6 & 82.8 & 83.6 \\
\cline { 3 - 6 } & & $\leq 7.5 \mathrm{~mm}$ & 88.5 & 79.3 & 83.6 \\
\hline Index size/depth & 0.931 & $\geq 1.54$ & 88.5 & 79.3 & 83.6 \\
\cline { 3 - 6 } & & $\geq 2.00$ & 80.8 & 82.8 & 81.8 \\
\hline Size of the tumor & 0.674 & & & & \\
\hline
\end{tabular}

such extensive calculations are not necessary, though the mentioned paper showed a potentially interesting and reliable way of tumor assessment. More suitable for routine clinical use than Demmy's formulas is the linear function (depth $=0.836 \times$ size 2.811) presented by Saito et al. [20]. Our index "size/depth" with a cut-off value $\geq 1.54$ produced similar results in prediction of successful localization of a tumor, though, and it is easier to calculate.

Detection of an occult tumor may be specific to each individual. Sometimes the tumor location in the vicinity of a main bronchus or other structure can make this simple identification impossible. It also requires extensive experience in minimally invasive thoracic surgery because this videothoracoscopic "palpation" must be correctly interpreted in order to recognize a pathological finding from a normal one. In our study this was not an issue because all procedures were performed by experienced chest surgeons with a history of hundreds of performed minimally invasive thoracic procedures.

When videothoracoscopic instrument-only detection of a lung nodule fails it does not mean clearly that it would be necessary to make a standard thoracotomy. It is usually possible to simply increase the size of one of the trocar incisions and insert a finger through. With the assistance of an instrument pushing the lung tissue against the finger it is possible to find the majority of occult lesions. In our experience and in correlation with the results of other authors, the frequency of thoracotomies due to the inability to find a lesion in another way is relatively low, and moreover, there are still some doubts as to whether it may significantly increase the sensitivity of detection of occult lesions [21]. The clinical impact of an increase in incision length is not as significant as it may seem. Pain is sometimes even more intense after videothoracoscopy due to bruis- ing of the intercostal space by a trocar and the negative influence of mechanics of ventilation is also generally not substantial $[9,22]$. In any case, if detection of a tumor just by using videothoracoscopic instrument-only "palpation" fails, a possibility is the perioperative use of endoscopic ultrasound examination, which may help localize the lesion without the necessity of special preoperative management [23-25].

When we expect trouble in the detection of certain occult lesions it is still possible to prepare a patient for the procedure and employ a special technique to label the tumor site. Under CT navigation it is possible to introduce a hook or a coil into the tissue adjacent to the tumor or into the tumor directly. Another possibility is to apply dye or radiotracer detected with radio-probe perioperatively. It may increase the success in the detection of occult lesions via the videothoracoscopic approach [12, 13, 24]; on the other hand, these methods are expensive and/or are related to some possible complications (mainly pneumothorax). Using radiotracer would also require special management of a patient due to the use of a radioactive material.

\section{Conclusions}

Evaluation of objective CT measurements may help predict the probability of detection of chondromatous hamartomas or other occult lesions of the lung using the videothoracoscopic approach and as such facilitate the decision of whether it is necessary to use a special technique instead. In our data, the main predictors are considered to be the depth of the tumor location in the lung parenchyma with a cut-off value $\leq 7.5 \mathrm{~mm}$ and the index "size/depth" with a cut-off value $\geq 1.54$. The tumor size itself does not seem to be a good predictor for this purpose. 


\section{References}

1. Jacobaeus HC. Possibility of the use cystoscope for investigation of serous cavities. Munch Med Wochenschr 1910; 57: 2090-2.

2. Mack MJ, Aronoff RJ, Acuff TE, et al. Present role of thoracoscopy in diagnosis and treatment of diseases of the chest. Ann Thorac Surg 1992; 54: 403-9.

3. Duda M, Gryga A, Czudek S, Skalický P. Twenty years of minimally invasive surgery in the Czech Republic. Videosurgery Miniinv 2011; 6: 42-7.

4. Belák J, Kudláč M, Šimon R. Video-assisted thoracoscopic surgery lobectomy - early experience. Videosurgery Miniinv 2010; 5 100-3.

5. Śmigielski J, Piskorz L, Wawrzycki M, et al. Assessment of quality of life in patients with non-operated pancreatic cancer after videothoracoscopic splanchnicectomy. Videosurgery Miniinv 2011; 6: 132-7.

6. Stefaniak T, Pirski MI, Osęka T, et al. Simultaneous bilateral transthoracic sympathectomy through posterior access in LinTelaranta modification for primary hyperhidrosis. Videosurgery Miniinv 2009; 4: 47-52.

7. Giudicelli R, Thomas P, Lonjon T, et al. Video-assisted minithoracotomy versus muscle-sparing thoracotomy for performing lobectomy. Ann Thorac Surg 1994; 58: 712-8.

8. Nomori H, Horio H, Naruke T, Suemasu K. What is the advantage of thoracoscopic lobectomy over a limited thoracotomy procedure for lung cancer surgery? Ann Thorac Surg 2001; 72: 879-84.

9. Miller JD, Urschel JD, Cox G, et al. A randomized, controlled trial comparing thoracoscopy and limited thoracotomy for lung biopsy in interstitial lung disease. Ann Thoras Surg 2000; 70: 1647-50.

10. Gossot D. Totally thoracoscopic basilar segmentectomy. Semin Thorac Cardiovasc Surg 2011; 23: 67-72.

11. Kerrigan DC, Spence PA, Crittenden MD, Tripp MD. Methylene blue guidance for simplified resection of a lung lesion. Ann Thorac Surg 1992; 53: 163-4.

12. Mack MJ, Gordon MJ, Postma TW, et al. Percutaneous localization od pulmonary nodules for thoracoscopic lung resection. Ann Thorac Surg 1992; 53: 1123-4.

13. Mack MJ, Shennib H, Landreneau RJ, Hazelrigg SR. Techniques for localization of pulmonary nodules for thoracoscopic resection. J Thorac Cardiovasc Surg 1993; 106: 550-3.

14. Bateson EM. So-called hamartoma of the lung - a true neoplasm of fibrous connective tissue of the bronchi. Cancer 1973; 31: 1458-67.

15. Van den Bosch JM, Wagenaar SS, Elbers JRJ, et al. Mesenchymoma of the lung (so called hamartoma): a review of 154 parenchymal and endobronchial cases. Thorax 1987; 42: 790-3.

16. Takemura T, Kusafuka K, Fujiwara M, et al. An immunohistochemical study of the mesenchymal and epithelial components of pulmonary chondromatous hamartoma. Pathol Int 1999; 49: 938-46.

17. Basile A, Gregoris A, Antonci B, Romanelli M. Malignant change in a benign pulmonary hamartoma. Thorax 1989; 44: 232-3.

18. Tamura M, Oda M, Fujimori H, Shimizu Y, Matsumoto I, Watanabe G. New indication for preoperative marking of small peripheral pulmonary nodules in thoracoscopic surgery. Interact Cardiovasc Thorac Surg 2010; 11: 590-3.
19. Demmy TL, Wanger-Mann CC, James MA, et al. Feasibility of mathematical models to predict success in video-assisted thoracic surgery lung nodule excision. Am J Surg 1997; 174: 20-3.

20. Saito H, Minamiya Y, Matsuzaki I, et al. Indication of preoperative localization of small peripheral pulmonary nodules in thoracoscopic surgery. J Thorac Cardiovasc Surg 2002; 124: 1198-202.

21. Nakajima J, Murakawa T, Fukami T, et al. Is finger palpation at operation indispensable for pulmonary metastasectomy in colorectal cancer? Ann Thorac Surg 2007; 84: 1680-4.

22. Bernard A, Brondel L, Arnal E, Favre JP. Evaluation of respiratory muscle strength by randomized controlled trial comparing thoracoscopy, transaxillary thoracotomy and posterolateral thoracotomy for lung biopsy. Eur J Cardiothorac Surg 2006; 29: 596-600.

23. Mack MJ, Shennib H, Landreneau RJ, Hazelrigg SR. Techniques for localization of pulmonary nodules for thoracoscopic resection. J Thorac Cardiovasc Surg 1993; 106: 550-3.

24. Piolanti M, Coppola F, Papa S, Pilotti V, Mattioli S, Gavelli G. Ultrasonographic localization of occult pulmonary nobles during video-assisted thoracic surgery. Eur Radiol 2003; 13: 2358-64.

25. Daniel TM. A proposed diagnostic approach to the patient with subcentimeter pulmonary nodule: techniques that facilitate video-assisted thoracic surgery excision. Semin Thorac Cardiovasc Surg 2005; 17: 115-22.

Received: 4.04.2012, revised: 2.07.2012, accepted: 20.07.2012 